\title{
Update: A food-borne outbreak of hepatitis A in the Netherlands related to semi-dried tomatoes in oil, January-February 2010
}

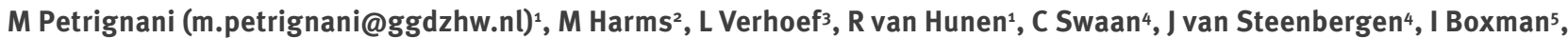

R Peran i Sala ${ }^{6}, \mathrm{H}_{\text {J Ober }}$, H Vennema ${ }^{3}$, M Koopmans $^{3}$, W van Pelt ${ }^{2}$

1. Department of Infectious Disease Control, Municipal Health Service (GGD Zuid-Holland West), Zoetermeer, the Netherlands

2. Epidemiology and Surveillance Unit, Centre for Infectious Disease Control, National Institute for Public Health and the Environment (Rijksinstituut voor Volksgezondheid en Milieu, RIVM), Bilthoven, the Netherlands

3. Laboratory for Infectious Diseases and Screening, Centre for Infectious Disease Control, National Institute for Public Health and the Environment (Rijksinstituut voor Volksgezondheid en Milieu, RIVM), Bilthoven, the Netherlands

4. Preparedness and Response Unit, Centre for Infectious Disease Control, National Institute for Public Health and the Environment (Rijksinstituut voor Volksgezondheid en Milieu, RIVM), Bilthoven, the Netherlands

5. Food and Consumer Product Safety Authority (Voedsel en Waren Autoriteit), Zutphen, the Netherlands

6. Food and Consumer Product Safety Authority (Voedsel en Waren Autoriteit), the Hague, the Netherlands

7. Food and Consumer Product Safety Authority (Voedsel en Waren Autoriteit), Amsterdam, the Netherlands

Citation style for this article:

Citation style for this article: Petrignani M, Harms M, Verhoef L, van Hunen R, Swaan C, van Steenbergen J, Boxman I, Peran i Sala R, Ober HJ, Vennema H, Koopmans $M$, van Pelt W. Update: A food-borne outbreak of hepatitis A in the Netherlands related to semi-dried tomatoes in oil, January-February 2010 . Euro Surveill.

2010;15(20): pii=19572. Available online: http://www.eurosurveillance.org/ViewArticle.aspx?Articleld=19572

This article has been published on 20 May 2010

Between 31 December 2009 and 10 February 2010, 13 patients were infected by an identical hepatitis $A$ virus strain not previously detected in the Netherlands. They had not been abroad and were widely distributed over the Netherlands. A case-control study including 12 cases and 44 controls identified semi-dried tomatoes in oil as the source of the outbreak (odds ratio: 20.0; $95 \%$ confidence interval: $1.5-274)$. The virus was not detected in any of 81 tested food samples. International trace-back is still ongoing.

\section{Introduction}

On 12 February 2010, five patients with acute hepatitis were detected in the Netherlands through our enhanced molecular surveillance programme and found to harbour an identical strain of hepatitis $A$ virus (HAV, Hu/Netherlands/RIVM-006/2010). These patients had not been abroad and did not cluster geographically. Although the number of reported HAV cases was normal for the time of the year, finding five identical HAV strains was unusual and triggered an outbreak investigation [1]. Because the nucleotide sequence of a fragment of the $\mathrm{VP}_{1-2} \mathrm{~A}$ region of HAV isolated from patients was identical to that found in patients involved in an outbreak in 2009 in Australia [2], and because the sequences were unique in the HAV database at our institute, a relation between these two outbreaks was suspected. The outbreak of hepatitis A in Australia was epidemiologically associated with the consumption of semi-dried tomatoes. To find a possible common source for the Dutch cluster, an investigation was conducted. The main goals were to identify any potential source among the food products consumed by the patients, specifically those containing semi-dried tomatoes. This article describes the results of the case-control study and food sample analysis.

\section{Methods}

\section{Case-control study}

The outbreak investigation focused on reported cases of hepatitis A with patients who contracted their infection in the Netherlands. Hepatitis A is notifiable in the Netherlands when a person has clinical symptoms of jaundice and/or fever combined with an elevated level of hepatitis A IgM in their serum (confirmed HAV patient) or combined with an epidemiological relation to a confirmed HAV patient (probable HAV patient). A nationwide project in which all laboratories were asked to send in the serum samples of patients for sequence analysis was already ongoing at the time of this outbreak [3].

A case-control study was initiated. Cases were defined as all reported persons with hepatitis $A$ infection between 10 December 2009 (week 50) and 13 April 2010 (week 15) confirmed to have a primary infection with a genotype $1 \mathrm{~B}$ HAV strain with identical sequence in a $460 \mathrm{nt}$ fragment of the VP1-2A part of the genome, $\mathrm{Hu} /$ Netherlands/RIVM-006/2010 [4]. Cases related to primary cases and with onset of disease two weeks or more after the primary case were regarded as secondary cases. All primary cases were approached for inclusion.

To facilitate rapid source identification, controls were selected by three methods. The first group consisted of unrelated hepatitis A cases with onset of illness in the same time period. These unrelated cases were people who contracted their infection in the Netherlands, 
but had a different HAV strain or were epidemiologically related to patients confirmed to be infected with a different strain. The second group of controls were found among non-household contacts of the cases and a third group by taking a sample in the same geographical area and of the same age range as the cases. Partners and family members were excluded to be controls since they could have been immunised as part of the outbreak control activities.

The cases and non-household contacts were asked to answer a web-based or telephone questionnaire. The controls that were selected from the same geographical region got the questionnaire and a letter asking for their participation in the study sent by post.

The questionnaire covered personal information such as age, gender, place of residence, country of birth, vaccine status, symptoms, contact with other infected persons, and a detailed food history. The food history specifically included fresh produce and fruits that are eaten unpeeled and/or uncooked (including: cabbage lettuce, iceberg lettuce, other lettuce, raw spinach, raw endives, other raw vegetables, sandwiches, dried tomatoes, semi-dried tomatoes in oil, tapenade, raspberries, blackberries, berries, strawberries, dates, figs and other fruit), as well as shellfish and other products that are normally eaten uncooked (clams, oysters, other shellfish, other raw products and other ready-made products). The participants were asked to indicate the degree of certainty of their answers as 'surely', 'possibly', 'not' or 'don't remember'. They were asked to name the places where the products were purchased and where they normally went shopping.

Because the power of analysis of the different control groups separately was limited due to low numbers of cases and controls, all three control groups were combined. Data were analysed univariately with a Fisher's exact test for non-random association between factors, the corresponding odds ratio was adjusted for age, sex and the different control groups, using appropriate dummy variables. After univariate testing all variables with a $p$ value under 0,2 were used in a forward stepwise selection method to fit a multivariate model. Analyses were done in $\mathrm{R}$ (version 2.10).

\section{FIGURE}

Cases of hepatitis A contracted in the Netherlands between 10 December 2009 and 13 April 2010 (n=66)

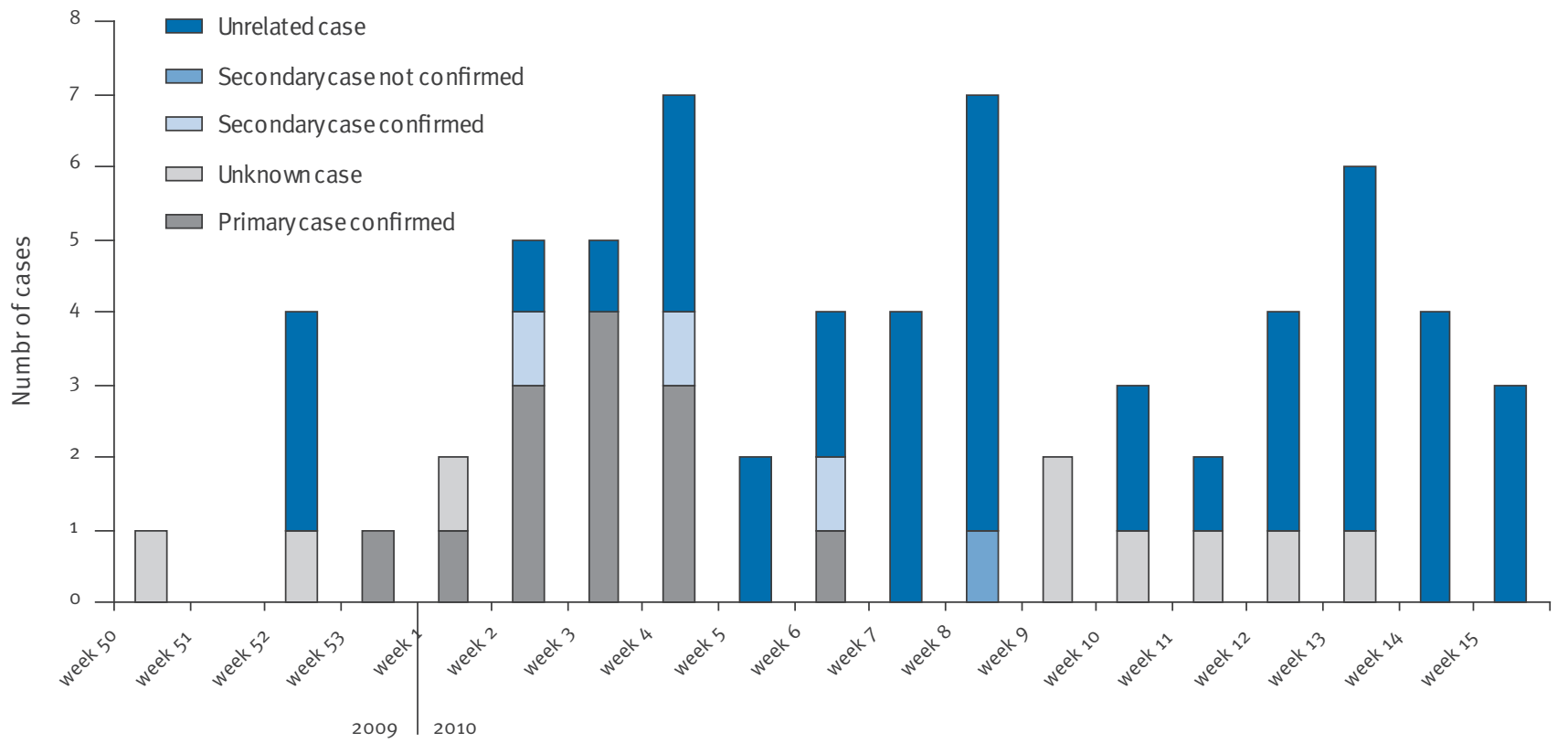

Onset of disease per week

TABLE

Odds ratios of risk factor for infection with the HU/Netherlands/RIVM-006/2010 strain in the Netherlands, 10 December 2009 - 13 April $2010(n=56)$

\begin{tabular}{|c|c|c|c|c|c|c|c|}
\hline \multirow{2}{*}{ Consumed semi-dried tomatoes in oil } & \multicolumn{2}{|c|}{ Total respondents } & \multicolumn{2}{|r|}{ Cases } & \multicolumn{2}{|c|}{ Controls } & \multirow[b]{2}{*}{ Odds ratio $^{\mathrm{a}}$} \\
\hline & $\mathbf{n}$ & Percentage & $\mathbf{n}$ & Percentage & $\mathbf{n}$ & Percentage & \\
\hline Not & 24 & $43 \%$ & 2 & $4 \%$ & 22 & $39 \%$ & 1.0 \\
\hline Surely and possibly & 22 & $39 \%$ & 8 & $14 \%$ & 14 & $25 \%$ & $20.0(1.5-274.1)$ \\
\hline Missing information & 10 & $18 \%$ & 2 & $4 \%$ & 8 & $14 \%$ & $11.6(0.4-312)$ \\
\hline Total & 56 & $100 \%$ & 12 & $22 \%$ & 44 & $78 \%$ & \\
\hline
\end{tabular}

${ }^{a}$ Odds ratio adjusted for age, sex and control groups. 
Source tracing and sampling

for laboratory analyses

Semi-dried tomatoes were implicated from the beginning of the investigation because the same strain was identified in an outbreak in Australia linked to semi-dried tomatoes [2]. In order to identify a common source of the semi-dried tomatoes consumed by the patients, the Food and Consumer Product Safety Authority performed trace back investigation on national and international suppliers of semi-dried tomato products. International trace-back information was exchanged via the Rapid Alert System for Food and Feed (RASFF) and Infosan. Initially, only little information was available on the brands of the products containing semi-dried tomatoes that had been consumed by the patients. For this reason, inspectors of the Food and Consumer Product Safety Authority collected a wide range of products at the stores used by the patients, and when national trace back information became available, samples were taken at the identified suppliers or warehouses located in the Netherlands.

All samples were analysed for the presence of HAV RNA at least in duplicate using an in-house method including an extraction process control and an external HAV RNA standard to control for inhibition of the PCR signal.

\section{Results}

\section{Background information}

As of 21 April 2010, a total of 66 cases were notified that had contracted their HAV infection in the Netherlands (Figure). Of these, 13 primary cases were confirmed to be infected by the same strain with onset of disease between 31 December 2009 and 10 February 2010 (one of them a British tourist). Four secondary cases were reported to be epidemiologically related to the confirmed primary cases (partners and family members), three of whom were infected by the Hu/Netherlands/ RIVM-006/2010 strain. For the fourth contact no serum was obtained for sequencing. Of the 13 confirmed primary cases, eight were male and five were female, with a median age of 42 years (range: 20-63 years). Nine cases with onset of disease between 10 December 2009 and 2 April 2010 and unknown transmission route could not be genotyped due to negative RT-PCR $(n=4)$ or lack of serum samples $(n=5)$ and were considered as 'unknown' cases. Of these nine unknown cases four were male and five were female, with a median age of 44 years (range: 6-69 years).

In the same period, 40 unrelated cases were reported who also contracted their infection in the Netherlands, but either had a different HAV strain or were epidemiologically related to patients confirmed to be infected by a different strain. Of the unrelated cases 16 were male and 24 were female, with a median of 20 years (range: 2-69 years).

\section{Case-control study}

13 primary confirmed cases and 262 controls were approached for inclusion. One case did not agree to participate in the study for unknown reasons. In total 61 controls filled out the questionnaire. Of the responding controls, 17 were excluded for returning an incomplete questionnaire. All further analysis was done based on a sample of 12 primary cases and 44 controls of which 12 were unrelated cases, 10 were non-household contacts of the patients and 22 were selected from the same geographical area.

For the univariate analysis the answers 'surely' and 'possibly' were taken together and 'don't remember' was classed as missing. Odds ratios were adjusted for age, sex and possible differences between control groups by adding two dummy variables to the model, which made it possible to differentiate between the three different control groups and see whether any of the groups had an effect on the model individually.

Univariately three variables showed a $p$ value of less than 0.2: dates, raw vegetables and semi-dried tomatoes in oil. Of these variables, dates were a protective factor and after multivariate analysis only semi-dried tomatoes in oil turned out to significantly improve the null model. Of the 10 respondents who answered this question, eight $(80 \%)$ confirmed having consumed semi-dried tomatoes in oil, compared to 14 of the 36 controls (39\%), giving an adjusted odds ratio of 20.0 (95\% confidence interval: 1.5-274.1) (Table).

Eight of the cases confirmed eating semi-dried tomatoes in oil, two did not remember and two denied having eaten them. Of these last four cases, two ate mixed salads that very likely contained semi-dried tomatoes, one case had surely consumed dry semi-dried tomatoes and the remaining case also had consumed mixed salads and often ate take-away food from restaurants.

\section{Source tracing and analyses of} semi-dried tomato products

In total 81 samples were collected between 23 February and 1 April 2010. These were semi-dried tomatoes in oil either marinated or not $(n=36)$, dried tomatoes without oil $(n=17)$, marinated semi-dried tomatoes $(n=16)$, tapenade or raw materials for tapenade containing semi-dried tomatoes $(n=20)$ and salads or dried salad mixes containing semi-dried tomatoes $(n=8)$. Five of these samples were collected as opened packages at the patients' homes (semi-dried tomatoes in oil $(n=2)$, dried tomatoes $(n=1)$, tapenade $(n=1)$ and dried salad mix containing semi-dried tomatoes $(n=1)$. In none of the 81 samples analysed, HAV RNA could be detected by two-step real-time RT-PCR. The products collected and specifically remembered by the patients were diverse and from different brands. Trace-back by Dutch food safety inspectors showed a complex product chain, involving multiple companies, and leading to international importers in different countries. Until now no common source could be identified, but we are still awaiting feedback from international trace-back through RASFF and Infosan. 


\section{Discussion}

The case-control study confirmed food products containing semi-dried tomatoes in oil as a risk factor for the hepatitis A cluster. Nearly all patients ate semidried tomatoes while only relatively few of the controls did. This is true for all three control groups and provides strong epidemiological evidence that semi-dried tomatoes were the source of the outbreak. Semi-dried tomatoes have not been described before as a cause of food-borne outbreaks of hepatitis A. When patients are asked what raw products they have consumed, it is likely that they will not mention semi-dried tomatoes spontaneously. Therefore we recommend keeping this product in mind when implementing a food questionnaire in the investigation of a suspected food-borne outbreak.

In our study, controls were not selected according to a standardised epidemiological study design, nor were all controls selected by the same method, which may have introduced selection bias. This choice was made for practical reasons to enable rapid conclusions for source tracing. As preliminary analysis of individual groups showed comparable results, we considered it justified to analyse the controls as one group to increase power.

Our results could not be confirmed by food testing as all samples tested negative for HAV RNA. Several factors associated with food analyses in general and with virus testing in particular may have lead to the negative test results. Food testing for viruses is not done routinely and there are at present no accepted validated routine methods available, but most importantly: only part of the samples were taken from the product type associated with the highest risk in our investigation and most likely these were collected too late. Often, leftovers from the batch implicated in a food-related event have been discarded by the time samples are being taken, as a consequence of the long incubation period of hepatitis $A$. In the present study, sampling started only from mid-February 2010, whereas many of the confirmed patients had probably been exposed to the source already in December 2009. Other factors in general are the potential non-homogeneous distribution of (low amounts of) virus in the food, low efficiency of the method to extract the virus from the food, co-isolation of inhibitory agents from the food that interfere with the test method, or insufficient sensitivity of the detection method.

Unfortunately, the products remembered by the patients were of diverse origin, involving many companies and requiring international trace-back. Around ten companies in different countries have been identified that supply (marinated) semi-dried tomatoes in oil to the stores where patients purchased their products. The source of this outbreak remains obscure, and given the diffuse pattern of distribution of cases is likely to be a contamination event higher up in the food production chain. One hypothesis could be that contaminated water was used during cultivation of the tomatoes as has been described for green onions [5].

We are still waiting for international responses through the RASFF and Infosan systems about possible relationships between the different companies. Therefore, a common source cannot be ruled out and we cannot be certain that the outbreak has ended. It is possible that some of the contaminated products have been frozen and not been consumed yet. HAV can survive for several months in frozen produce as has recently been described for spinach leaves, berries and herbs $[6,7]$. Alternatively, there could still be a risk of contamination at the farm where the tomatoes originated from. Therefore, surveillance through sequence analysis of patient sera will remain necessary for several months at least.

\section{Acknowledgements}

We would like to thank Geke Hägele and Nathalie te Loeke for analyses of the food samples, Jeroen Cremer and Bas van der Veer for technical assistance on human sera, Joanne Bruins, Jolanda Rensink and Marlies van Dam for assisting the outbreak team. Furthermore, we would like to thank the Municipal Health Services for their help in including the patients, the Food and Consumer Product Safety Authority food inspectors for product sample collection and their back office for technical assistance as well as the medical microbiological laboratories involved for sending in the patient sera.

\section{References}

1. Petrignani $M$, Verhoef $L$, van Hunen $R$, Swaan C, van Steenbergen J, Boxman I, et al. A possible foodborne outbreak of hepatitis A in the Netherlands, January-February 2010. Euro Surveill. 2010;15(11). pii: 19512. Available from: http://www. eurosurveillance.org/ViewArticle.aspx?Articleld=19512

2. Hepatitis A virus, semi-dried tomatoes - Australia (03): (Victoria). ProMED-mail [online]. Boston US: International Society for Infectious Diseases; 4 November 2009. Archive no. 20091104.3811. Available from: http://www.promedmail.org

3. van Steenbergen J, Petrignani M, Kroneman A, Koopmans M. [The molecular epidemiology of hepatitis A in The Netherlands; the usefulness of typing isolated viral strains] Ned Tijdschr Geneeskd. 2008;152(7):408 [Article in Dutch].

4. Stene-Johansen K, Tjon G, Schreier E, Bremer V, Bruisten S, Ngui SL, et al. Molecular epidemiological studies show that hepatitis $A$ virus is endemic among active homosexual men in Europe. J Med Virol. 2007;79(4):356-65.

5. Wheeler $C$ et al. An outbreak of hepatitis $A$ associated with green onions. N Engl J Med. 2005;353(9):890-7.

6. Shieh YC, Stewart DS, Laird DT. Survival of hepatitis A virus in spinach during low temperature storage. J Food Prot. 2009;72(11):2390-3.

7. Butot S, Putallaz T, Sánchez G. Effects of sanitation, freezing and frozen storage on enteric viruses in berries and herbs. Int J Food Microbiol. 2008;126(1-2):30-5. 